\title{
Left ventricular dysfunction after degenerative mitral valve repair: A question of better molecular targets or better surgical timing?
}

\author{
Jordan D. Miller, PhD, a,b and Rakesh M. Suri, MD, DPhil
}

\footnotetext{
From the Departments of ${ }^{\mathrm{a}}$ Surgery and ${ }^{\mathrm{b}}$ Physiology and BME, Mayo Clinic, Rochester, Minn; and the ${ }^{\mathrm{c}}$ Department of Thoracic and Cardiovascular Surgery, Cleveland Clinic Foundation, Cleveland, Ohio. Disclosures: Authors have nothing to disclose with regard to commercial support.

Received for publication July 4, 2016; accepted for publication July 6, 2016; available ahead of print Aug 11 , 2016.

Address for reprints: Jordan D. Miller, PhD, Department of Surgery, Department of Physiology \& BME, Mayo Clinic, 200 First St SW, Rochester, MN 55905, and Rakesh M. Suri, MD, DPhil, Department of Thoracic and Cardiovascular Surgery, Cleveland Clinic Foundation, 9500 Euclid Ave, J4-1, Cleveland, OH 44195 (E-mail: miller.jordan@mayo.edu or surir@ccf.org).

J Thorac Cardiovasc Surg 2016;152:1071-4

$0022-5223 / \$ 36.00$

Copyright (C) 2016 by The American Association for Thoracic Surgery

http://dx.doi.org/10.1016/j.jtcvs.2016.07.018
}

Isolated mitral valve regurgitation (MR) is a condition affecting nearly 2.5 million patients in the United States alone. Left untreated, MR results in progressive impairment of cardiac function, ultimately leading to development of heart failure. Although surgical repair of the valve in patients with symptoms who have compromised left ventricular function (ie, class I indication) may provide symptomatic relief for these patients but not restore their normal survival expectancy, a significant body of work suggests that surgical repair of the valve while patients are free of symptoms and retain normal left ventricular function (ie, class IIa indication) may bestow greater benefit by restoring normal life expectancy in the majority of cases.

Improvement in cardiac function can be variable after mitral valve repair surgery in patients with class I indications for surgery; however, experts remain perplexed by the observation that left ventricular function can decline in a subset of patients after surgical amelioration of valve regurgitation in patients with class IIa indications for surgery. Although substantial effort has been put toward understanding molecular mechanisms underlying pathophysiologic changes in the valve apparatus itself,, ${ }^{1,2}$ little is known regarding molecular changes evident in the left ventricles of patients with preserved left ventricular function undergoing valve repair surgery.

In this issue of the Journal, Ahmed and colleagues ${ }^{3}$ report results obtained by a multidisciplinary team of surgeons, pathologists, biologists, and engineers who have been assembled by Dell'Italia to begin to probe molecular changes that may underlie susceptibility to postoperative declines in cardiac function in patients with isolated MR. First, through the use of magnetic resonance imaging with tissue tagging to quantify changes in cardiac function in subjects undergoing mitral repair surgery, Ahmed and

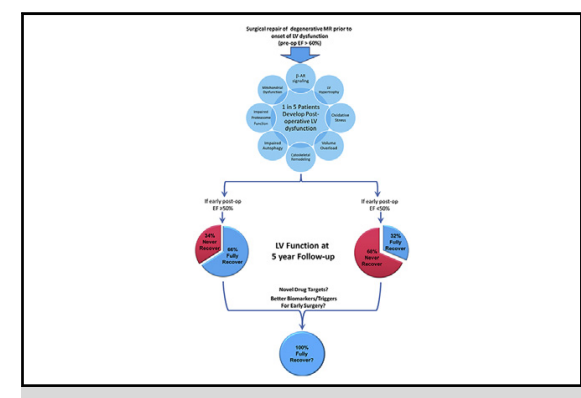

Prevalence and potential mechanisms of postoperative left ventricular dysfunction.

Central Message

Early repair of degenerative mitral valve regurgitation is important to prevent postoperative left ventricular dysfunction.

See Article page 1059.

colleagues $^{3}$ unequivocally demonstrated that systolic cardiac function was well preserved in before surgery and clearly showed that a subset of these subjects had impaired cardiac function at 6 postoperative months. This observation is entirely consistent with previous observations. ${ }^{4-6}$

Second, and critically, at the time of surgical mitral valve repair, surgeons removed a small section of ventricular tissue for histopathologic and molecular analyses. Starting with transmission electron microscopy, Ahmed and colleagues $^{3}$ showed severe disruption of the cytoskeletalmitochondrial architecture in cardiomyocytes from patients with MR, along with evidence of lipid droplet accumulation within the myocardium. Critically, this observation is consistent with recent work suggesting that impaired mitochondrial fission-fusion dynamics contribute to lipid accumulation and aberrant calcium signaling in the failing heart. $^{7,8}$

To delve more deeply into mechanisms underlying the abnormal localization and morphology of mitochondria in the volume-overloaded but compensated left ventricle, Dell'Italia's group ${ }^{3}$ used immunohistochemical approaches to evaluate changes in localization of desmin - a key protein dictating cellular architecture and organization-in cardiomyocytes from normal ventricles and the ventricles of subjects with severe MR. Importantly, they not only found evidence of reductions in total cellular desmin content in patients with MR but also found that desmin localization 


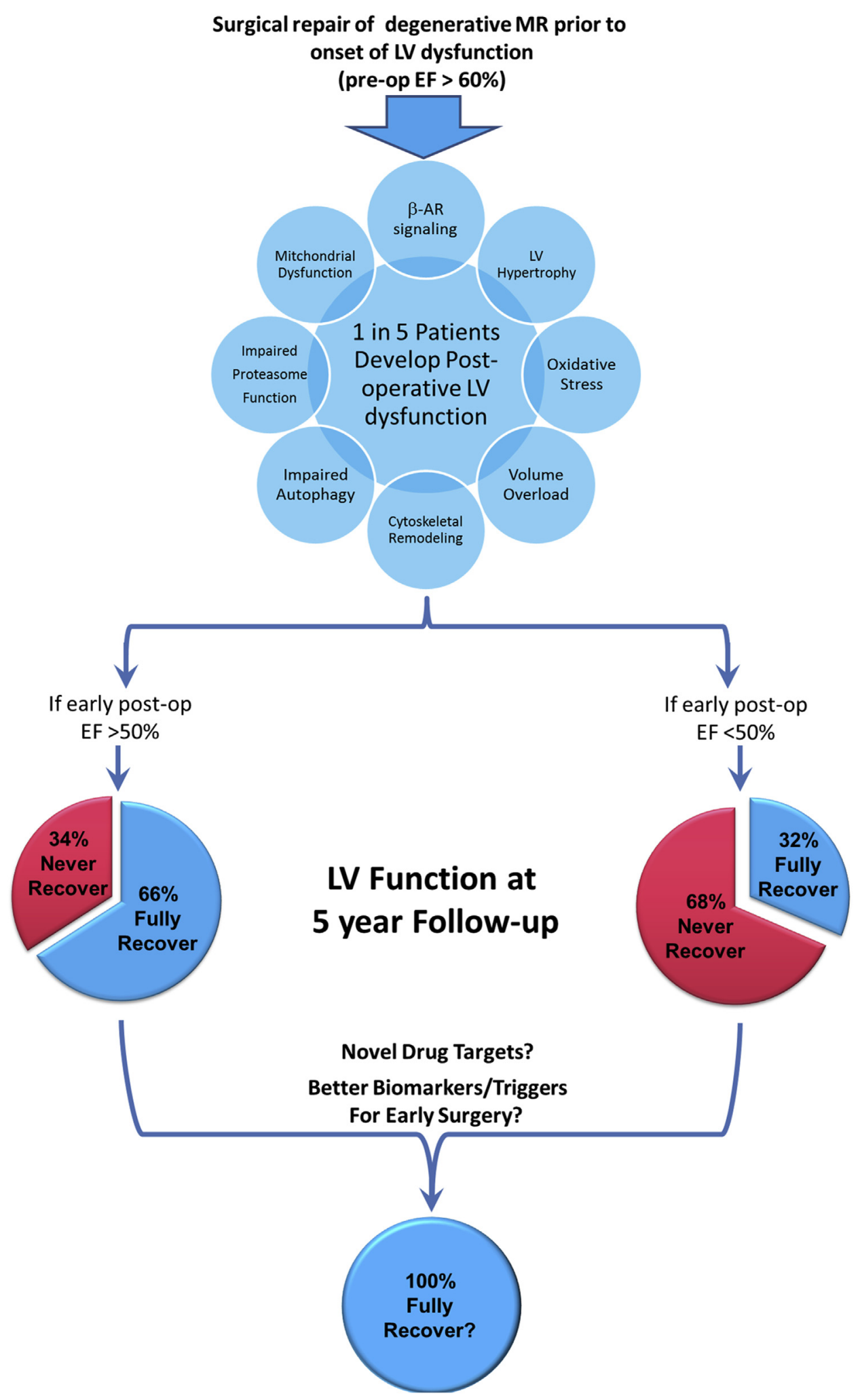

FIGURE 1. Prevalence and potential mechanisms of postoperative left ventricular ( $L V$ ) dysfunction. Note that roughly $20 \%$ of patients develop postoperative LV dysfunction, with the magnitude of that impairment being predictive of the likelihood of recovery of function. While numerous mechanisms have been proposed to underlie the transient impairment of LV function and its return to preoperative levels, few have been tested experimentally. Consequently, further research using experimental model systems to identify druggable molecular targets combined with multi-site, large-scale clinical studies to identify suitable biomarkers to trigger early intervention will be key to improving postoperative outcomes for all patients undergoing mitral valve repair surgery. $M R$, Mitral regurgitation; $E F$, ejection fraction; $\beta$ - $A R, \beta$-adrenergic receptor. 
patterns-which typically concentrate in a linear pattern with Z-disks and intercalated disks-formed perinuclear aggregates in cardiomyocytes from patients with MR. The aberrantly localized desmin aggregates also localized with the chaperone molecule $\alpha \beta$-crystallin, suggesting both that this is a regulated process and that there is a failure to clear cytoskeletal breakdown products. These findings align remarkably well with previous reports of abnormal mitochondrial morphology in hearts from desmin-deficient mice. $^{9}$

Finally, Ahmed and colleagues ${ }^{3}$ characterized changes in oxidative stress and antioxidant enzymes in ventricular tissues from patients with MR and found increases in 4hydroxynonenal - a highly reactive molecule generated by lipid peroxidation reactions in a number of cardiac diseases. ${ }^{10}$ Furthermore, increases in 4-hydroxynonenal were associated with an apparently compensatory increase in catalase levels that often associated with perinuclear desmin aggregates.

Collectively, the study by Ahmed and colleagues ${ }^{3}$ has provided us an initial glimpse into the molecular changes that occur in the myocardium of patients with severe MR. Although limited in scope, these findings suggest that the preservation of gross left ventricular systolic function is a poor indicator of preserved molecular processes. Like any good study, however, the work by Ahmed and colleagues ${ }^{3}$ raises more questions than it answers. What is the upstream driver of these molecular changes? Which changes are viable drug targets that drive the development of cardiac dysfunction after surgery, and which are readily reversible epiphenomena that are simply associated with severe MR? When placed in the context of other work, ${ }^{11,12}$ it is attractive to postulate that $\beta$-adrenergic receptor stimulation could be a key driver of increases in oxidative stress, reduced nitric oxide bioavailability, abnormal mitochondrial function, and impaired autophagy or mitophagy (Figure 1). Reciprocally, it is possible that volume overload per se is a primary driver of derangements in cytoskeletal structure, which subsequently initiate a cascade of events driving mitochondrial dysfunction, mitochondria-derived reactive oxygen species, and ultimately susceptibility to postoperative left ventricular dysfunction.

This study by Ahmed and colleagues ${ }^{3}$ confirms findings from recent studies from our group exposing the existence of a subpopulation of patients who have left ventricular impairment develop early after mitral valve repair. ${ }^{4-6}$ In a similar homogeneous population of patients with degenerative MR and preserved left ventricular function (ejection fraction $>60 \%$ ), we surprisingly discovered that approximately 1 in 5 patients had early left ventricular dysfunction (ejection fraction $<50 \%$ ) develop, and that among these, $20 \%$ were free of typical class I or class IIA indications before surgery. Perhaps the greatest clinical concern stemming from our study was the discovery that only 1 in 3 patients with postoperative left ventricular impairment had demonstrated ejection fraction recovery 5 years after correction of MR. Although reductions in ejection fraction of 5\% to $10 \%$ after mitral valve repair have heretofore been explained as a volumetric adjustment to the elimination MR, hypotheses to explain more significant degrees of left ventricular impairment have included myocardial fibrosis, hypertrophy, and adverse geometric remodeling. This work by Ahmed and colleagues ${ }^{3}$ dramatically increases the number of potential mechanisms contributing to postoperative LV dysfunction to include derangements in the cytoskeleton, mitochondria, and phagocytic and proteasomal pathways within cardiomyocytes.

Where do these lines of evidence take us as we face the patient with severe degenerative MR looking for answers? Mitral valve repair to correct severe degenerative MR is supported by science as safe, highly certain, and durable. ${ }^{13}$ Methods of characterizing a patient's predisposition to the development of left ventricular dysfunction, on the other hand, is still a pseudoscience at best. The report of Ahmed and colleagues ${ }^{3}$ provides a first glimpse into a scientific foundation for this phenomenon; it remains unclear, however, whether the magnitude of molecular derangements at the time of surgery is predictive of recovery of ventricular function in larger cohorts of patients. Furthermore, means of assessing this on a patient-specific basis has not matured to the point that it could guide clinicians with regard to the timing of surgical therapy. While the search for a viable diagnostic means of assessing molecular changes and targets amenable to drug therapy proceeds, prompt correction of degenerative MR, with $99 \%$ certainty and less than $0.3 \%$ risk, remains the best means of preserving myocardial function, allowing patients to return to a normal life, free of symptoms and with normal life expectancy. ${ }^{14}$

\section{References}

1. Levine RA, Hagége AA, Judge DP, Padala M, Dal-Bianco JP, Aikawa E, et al Mitral valve disease-morphology and mechanisms; Leducq Mitral Transatlantic Network. Nat Reviews Cardiol. 2015;12:689-710.

2. Thalji NM, Hagler MA, Zhang H, Casaclang-Verzosa G, Nair AA, Suri RM, et al. Nonbiased molecular screening identifies novel molecular regulators of fibrogenic and proliferative signaling in myxomatous mitral valve disease. Circ Cardiovasc Genet. 2015;8:516-28.

3. Ahmed MI, Guichard JL, Rajasekaran NS, Ahmad S, Mariappan N, Litovsky S, et al. Disruption of desmin-mitochondrial architecture in patients with regurgitant mitral valves and preserved ventricular function. J Thorac Cardiovasc Surg. 2016;152:1059-70.e2.

4. Quintana E, Suri RM, Thalji NM, Daly RC, Dearani JA, Burkhart HM, et al. Left ventricular dysfunction after mitral valve repair - the fallacy of "normal" preoperative myocardial function. J Thorac Cardiovasc Surg. 2014;148:2752-60.

5. Suri RM, Schaff HV, Dearani JA, Sundt TM, Daly RC, Mullany CJ, et al. Recovery of left ventricular function after surgical correction of mitral regurgitation caused by leaflet prolapse. J Thorac Cardiovasc Surg. 2009;137:1071-6.

6. Suri RM, Schaff HV, Dearani JA, Sundt TM III, Daly RC, Mullany CJ, et al. Determinants of early decline in ejection fraction after surgical correction of mitral regurgitation. J Thorac Cardiovasc Surg. 2008;136:442-7.

7. Hom J, Sheu SS. Morphological dynamics of mitochondria — a special emphasis on cardiac muscle cells. J Mol Cell Cardiol. 2009;46:811-20.

8. Yang S, Xia C, Li S, Du L, Zhang L, Zhou R. Defective mitophagy driven by dysregulation of rheb and KIF5B contributes to mitochondrial reactive oxygen 
species (ROS)-induced nod-like receptor 3 (NLRP3) dependent proinflammatory response and aggravates lipotoxicity. Redox Biol. 2014;3:63-71.

9. Capetanaki Y. Desmin cytoskeleton: a potential regulator of muscle mitochondrial behavior and function. Trends Cardiovasc Med. 2002;12:339-48.

10. Mali VR, Palaniyandi SS. Regulation and therapeutic strategies of 4-hydroxy-2nonenal metabolism in heart disease. Free Radic Res. 2014;48:251-63.

11. Trappanese DM, Liu Y, McCormick RC, Cannavo A, Nanayakkara G, Baskharoun MM, et al. Chronic $\beta 1$-adrenergic blockade enhances myocardial $\beta 3$-adrenergic coupling with nitric oxide-cGMP signaling in a canine model of chronic volume overload: new insight into mechanisms of cardiac benefit with selective $\beta 1$-blocker therapy. Basic Res Cardiol. 2015;110:456.
12. Nakamura K, Kusano K, Nakamura Y, Kakishita M, Ohta K, Nagase S, et al. Carvedilol decreases elevated oxidative stress in human failing myocardium. Circulation. 2002;105:2867-71.

13. Suri RM, Taggarse A, Burkhart HM, Daly RC, Mauermann W, Nishimura RA, et al. Robotic mitral valve repair for simple and complex degenerative disease: midterm clinical and echocardiographic quality outcomes. Circulation. 2015; 132:1961-8.

14. Suri RM, Vanoverschelde JL, Grigioni F, Schaff HV, Tribouilloy C, Avierinos JF, et al. Association between early surgical intervention vs watchful waiting and outcomes for mitral regurgitation due to flail mitral valve leaflets. JAMA. 2013;310:609-16. 